\title{
Implementation of an educational model leveraging the power of incentive reward theory with Blockchain.
}

\author{
Rishi Gulati ${ }^{1, *}$, Akash Jethi ${ }^{1,}$, Arnab Ghosh ${ }^{1,}$, Abhishek Shimpi ${ }^{1,}$, and Ashwini Naik ${ }^{2}$, \\ ${ }^{1}$ U.G. Student, Department of Electronics and Telecommunication, Ramrao Adik Institute of Technology, Navi Mumbai, Maharashtra, \\ India. \\ ${ }^{2}$ Professor, Department of Electronics and Telecommunication, Ramrao Adik Institute of Technology, Navi Mumbai, Maharashtra, \\ India
}

\begin{abstract}
Recent studies conducted to investigate the reasons for an alarming decline in the performance of undergraduate students have thrown up a few compelling facts. These necessitate our immediate attention. Demographic shuffling along with lack of intrinsic motivation stand out as the cardinal reasons among other miscellaneous ones. There is a need to introduce programs that perk up one's persistence level and provide the missing tangible incentive for a purposeful engagement with our education system. The paper endeavours to fulfil this requirement through the use of Blockchain (cryptocurrency) guided by the principles of extrinsic motivation theory.
\end{abstract}

\section{Introduction}

Problem solving is commonly understood as tweaking what is wrong but an alternate approach that seeks to rectify by over emphasizing or amplifying what's right can at times be cleverly adopted. Methodology for solving a problem faced by Universities tends on similar lines. It advocates the use of Reward based Learning coupled with Aversion Theory to achieve an improved student performance.

In this approach incentives are offered to lure every individual to emulate appreciable behavioural traits. This paper proposes the use of public/ private cryptocurrency, says an ether/RAIT Coin with its scope global/ restricted inside the precincts of the University, as a reward for exemplary behaviour displayed by the students. A set of scenarios have been thought and suggested where cryptocurrency can be earned by the students while short listing the services where they could also be spent. We have further incorporated the doctrine of punishment through payment of fines in case of noncompliance by the students in our scheme of things. Students will also be able to continually monitor their rewards earned in their "Wallets" created via a webapplication. All this will positively help Universities in improving their student profiles and abate the continued pressure felt by them from state legislatures, broader public and their own placement partners to become more accountable for the performance of their students.

This paper focuses on the root concepts and their implementation to integrate the brand-new advanced model over the existing traditional model. The paper proposes a course of action for the appliance of the improved model

\section{Root Concepts}

\subsection{Broadening the niche}

The philosophy of rewarding the students for exemplary behaviour is already adopted by a few universities. These schemes have often targeted the cream students (E.g. A cash reward for the top five rank holders). This approach fizzles out and bares minimal success as it tempts to a very niche group of students and fails to captivate the interest of the average student.

The paper proposes to replace this with a new ideology revolving around the concept of setting a list of goals in compliance with the institute's guidelines. The key is to have goals for every student which he/she believes to be achievable. This ensures that the scheme gets the mass of students involved and increases the probability of success.

\subsection{The stick carrot theory}

The stick carrot theory is a policy which explores the use of punishments along with rewards to recondition people to a fitting behaviour. The idea is to incorporate the issuance of fines as a lenient punishment and act as a negative reinforcement.

While the use of extrinsic motivation to earn rewards is the primary arrangement the motivation to avoid being issued bills (Deterrence strategy) is of equal importance. The following diagram illustrates a very basic overview of the proposed model

*e-mail: gulatirishi29@gmail.com 


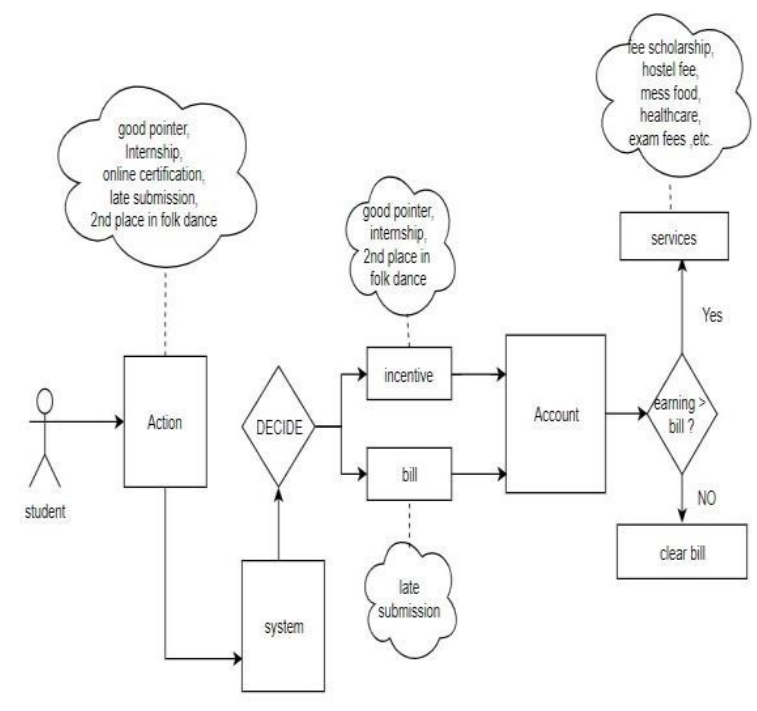

Figure 1. Overview of stick carrot model.

\subsection{The Decision Buffer}

The model must make some important decisions and uses specific algorithms for it. The first decision is to compare the student performance with fixed guidelines and decide upon issuing a bill or a reward. The second and the more important decision is to determine the value of bill/award to be issued.

A simpler approach would be to calculate the measure of shortcoming/ideal behaviour of student and use it to derive the value. The approach used here is a hybrid one which uses the value generated by simple approach and then modifies it depending on the modulation coefficient calculated by using the user's historical data. The coefficient value is dependent on the measure of variance between current data readings and historical data. Additional factors can be used to bias the decision.

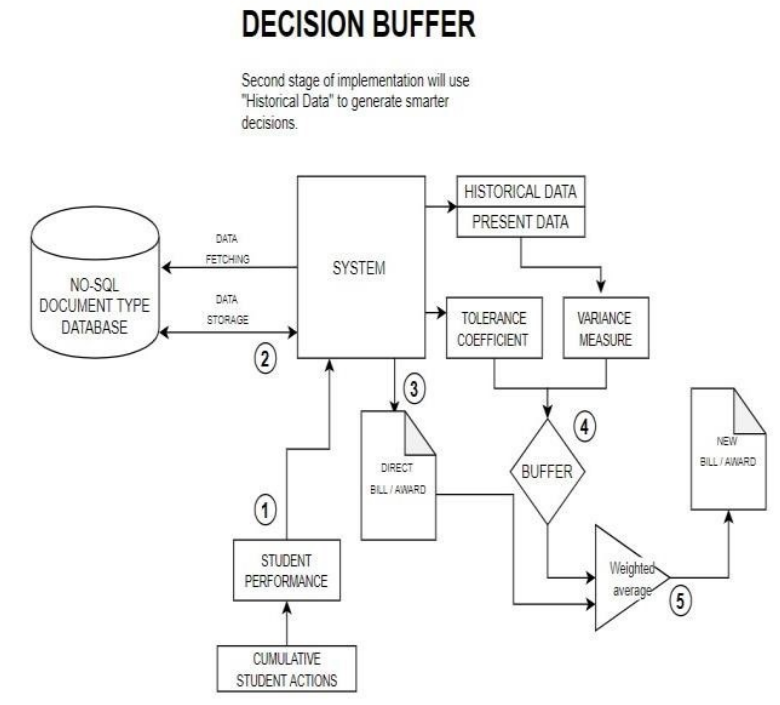

Figure 2. Overview of decision buffer system.

\subsection{The FPP}

The FFP is an acronym for the frequent flier program but in the model it stands for the free fund program. The free fund program is inspired from the FFP which rewards user with travel miles for using the airlines. These miles can be spent by the user in exchange for other services which they desire.

Similarly under the free fund program the students can use the cryptocurrency they are awarded in exchange for various services provided by the organization. In absence of such a perk program the user will be satisfied by earning the bare minimum required to clear the bills. The FPP plays a vital role in propelling the user to earn maximum cryptocurrency. Having certain services that can be availed exclusively only by the earned cryptocurrency will further motivate the users.

\subsection{The Light house effect}

The extrinsic motivation and deterrence strategy certainly work however they may not be enough. The efforts and effects of the model can be compounded by use of lighthouse effect. Just like a lighthouse guides ships in storm to safe harbours, the model uses a recommendation system to guide the students. After correlating the students' performance (shortcomings and merits) with the guidelines of the university the system will generate recommendation tailored to each user.

The system operates with aim of providing the students with a gentle push in the right direction. Example of a typical recommendation generated for a student "ABC" may be 'To attend two of next three lectures of "XYZ". ' subject in the coming week as this will bolster his/her attendance above $70 \%$ and he/she will be eligible for a reward.

The following diagrams illustrates the basic operation cycle of generation of all the insights and recommendations for the end user. 


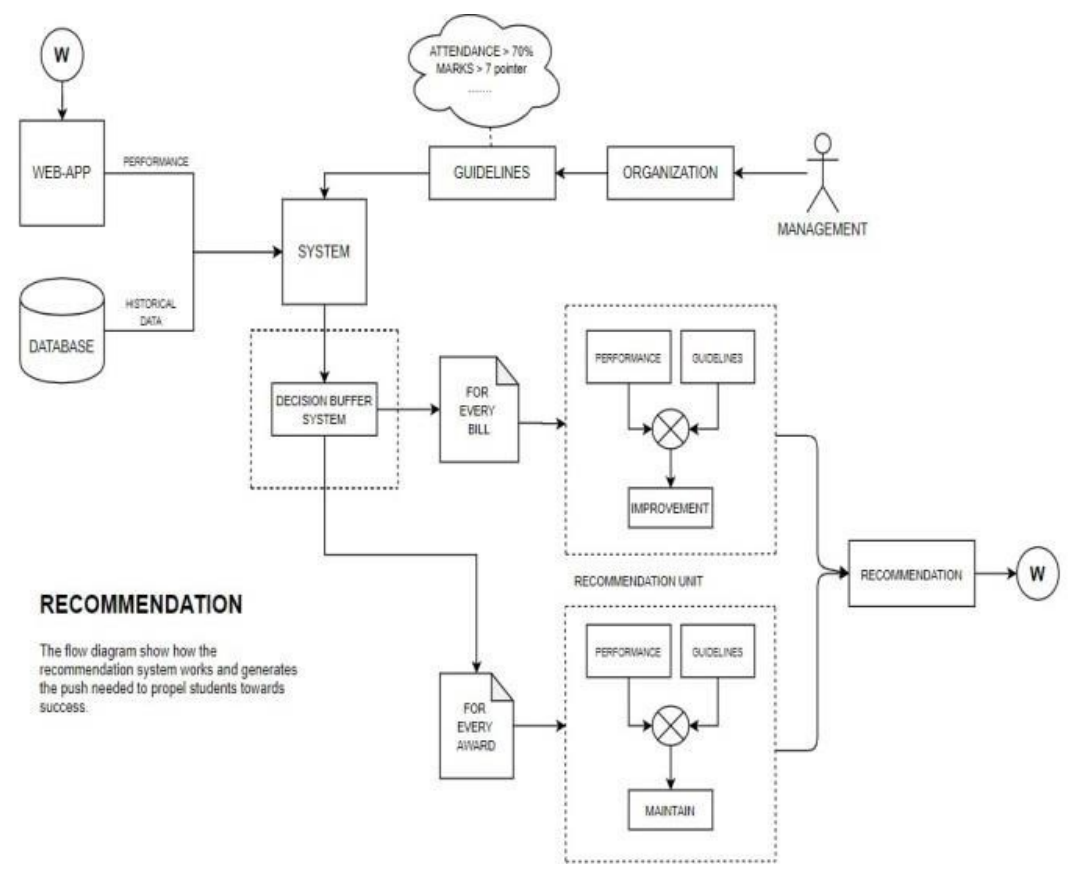

Figure 3. Overview of recommendation system

\subsection{Cibil score}

\subsubsection{Cibil as a performance metric}

Up until now the students academic success is only gauged their pointer. The pointer is a specific measure of marks scored and fails to give any other information about the student. Important parameters like attendance, timely submissions, involvement in extra-curricular activities and sports, individual achievements like winning a paper presentation competition often get neglected. The concept of maintain a Cibil score is inspired from the banks/insurance companies who maintain the credit activity of every individual to take calculated decisions. The model maintains a Cibil score with range 300 to 900 for every student which is incremented every time the student is rewarded and viceversa. Since the awards and bills are generated for every factor of the students' performance the Cibil score acts as an overall performance metric of the student.

\subsubsection{Cibil as a utility}

Just as in polymorphism the Cibil score is overloaded to have more than one function. Its primary function remains to be viewed as a performance metric. The first added function is derivation of defaulter list based on Cibil. The second added function is to convert The Cibil into a utility to motivate the students to maintain a higher Cibil score. This process is carried out in steps.

1. Calculate Cibil influence factor based on the degree of variance in Cibil score over a certain period.

2. Every purchasable service will offer a discount. The discount depends on the students Cibil influence factor.
3. Every Bill payment offers "Xamount of the bill paid. The cash back percentage depends on the Cibil score. 


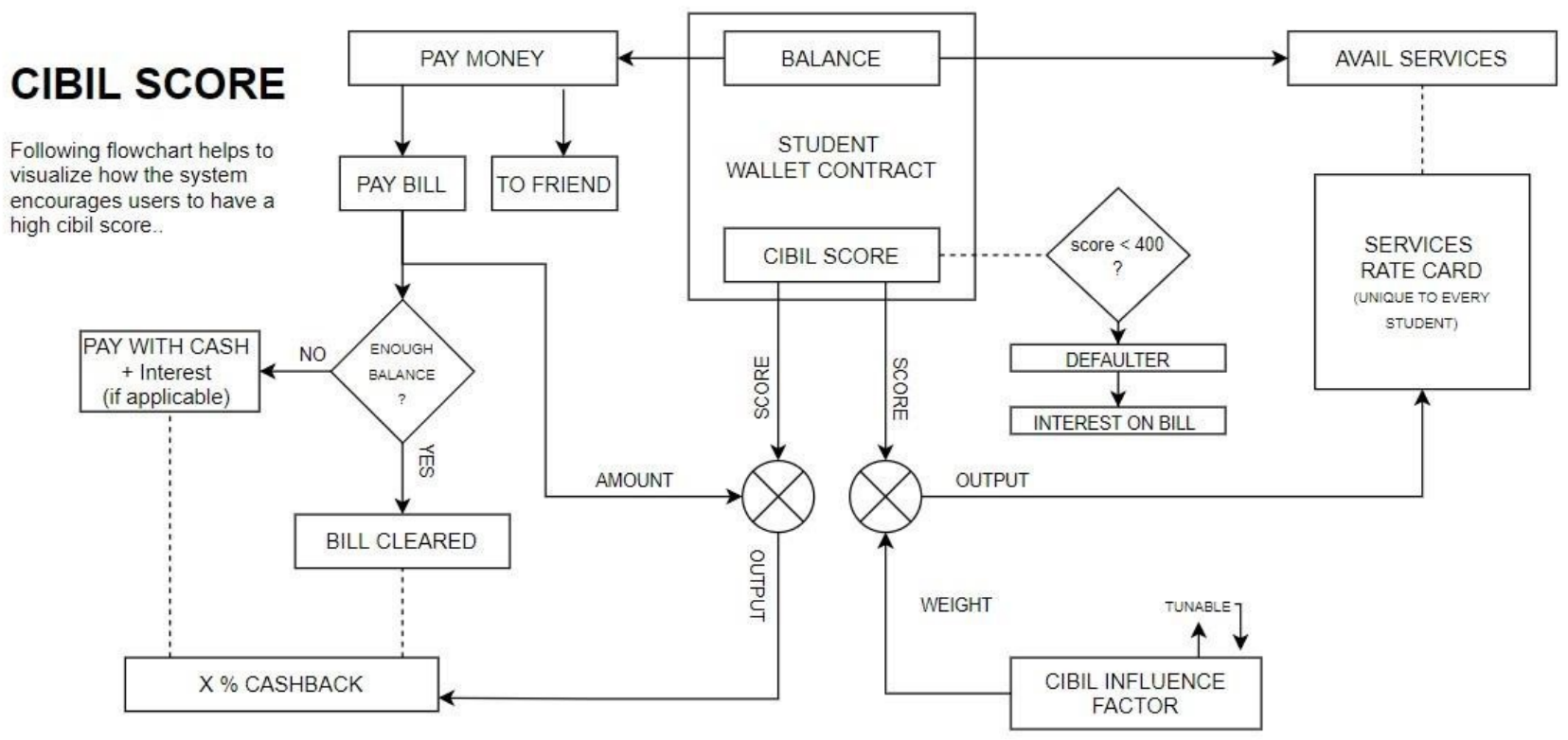

Figure 4. Overview of Cibil score's importance.

\section{The Implementation blueprint}

\subsection{Using public Ether}

If the organization decides to invest whole-heartedly in the model then a public coin like Ethereum can be used. The biggest pro of this approach is that the person being rewarded can use the reward outside the organization. The economics of this approach may make it unsuitable for many colleges. While some colleges have the resources to implement it; this approach is most suitable for commercial organizations to motivate their employees to gain advantage in today's cut-throat market. The users are expected to have a metamask account. The crypto-wallet in metamask is interfaced with web application. The system works with 3 contract types written in solidity.

The factory contract is a master contract used to deploy additional bank and wallet contracts on the Ethereum Blockchain. Every department has a bank contract assigned to it by the factory contracts constructor. On the other hand a new wallet contracts is deployed and assigned to the user by the factory each time a new user registers themselves. Each user's wallet contract has total earning, total bill, current balance and Cibil score associated with it. The transactions mostly take place between the bank and wallet contracts and are added to a block and mined on the main Ethereum network; therefore gas must be paid with each transaction. Every transaction takes about 25 seconds for completion. 


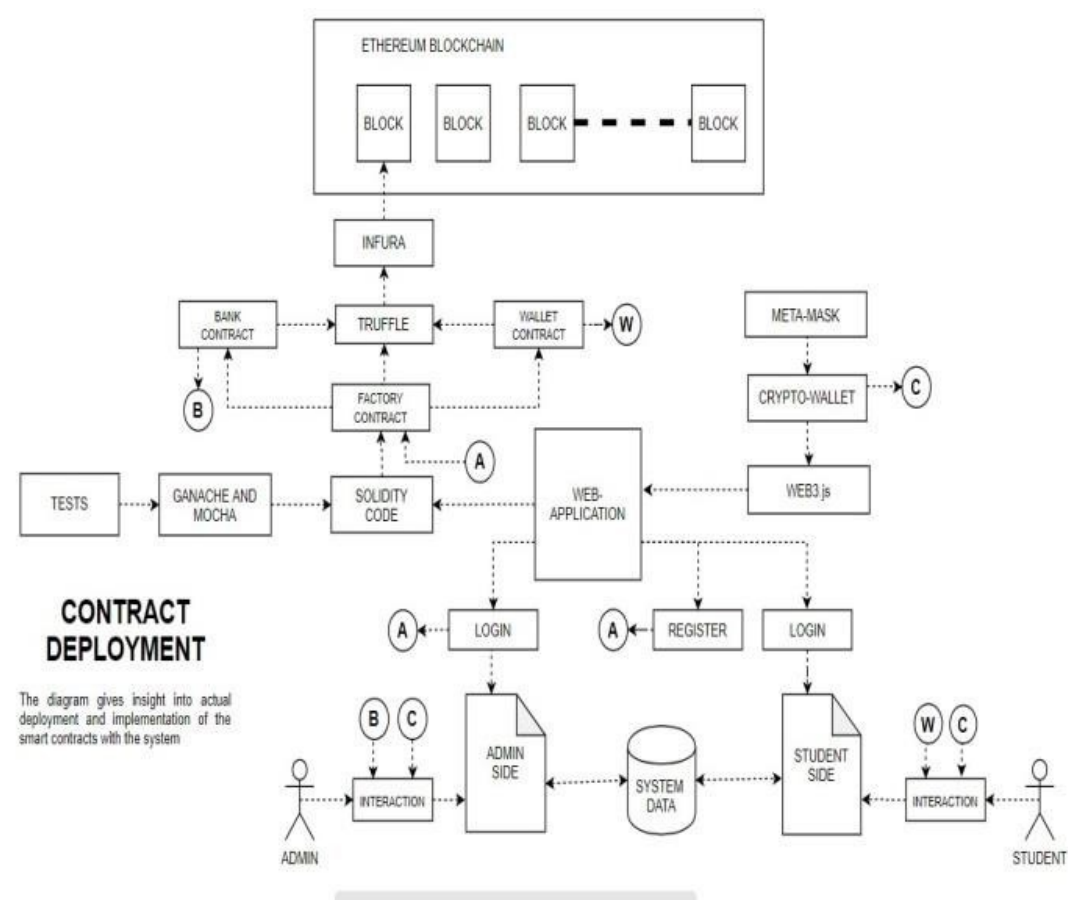

Figure 5. Public cryptocurrency blueprint

\subsection{Using private cryptocurrency}

For an organization with a lot of manpower (students/employees) it becomes difficult to reward with cryptocurrency because if there is a substantial difference in the currency awarded and currency recouped as bills it can drain the organization financially. To tackle this conundrum the paper proposes the creation of a private cryptocurrency with its scope restricted to the organization.

Though the rewards cannot be used outside the premises of the organization the low financial risk of this approach makes it suitable for universities and small organizations. The operational flow of student interacting with the portal remains explicitly same, though the process of making transactions strays from the original. The presence of a Metamask account which was mandatory in the first approach is no longer a necessity. The authentication and security of the whole process is monitored in three steps.

1. The verification of the transaction, relying heavily on the RSA algorithm. While sending funds the sender needs to digitally sign the transaction with their private key.

2. The authentication of the block, which involves matching the current hash of the block with previous hash. The miner involved in this process calculates the nonce and verifies the transactions by verifying the digital signature of the sender using their respective public key.

3. The authentication of the complete Blockchain. It is solely based on the concept of the consensus theory.

Private coin allows tinkering with the gas which is to be rewarded to miners. The model advocates two ap- proaches. First approach suggests that the organization will mine every transaction involving the private coin. The second approach dictates that each individual must mine his own transactions; this approach eliminates the sending of additional gas along with transactions. 


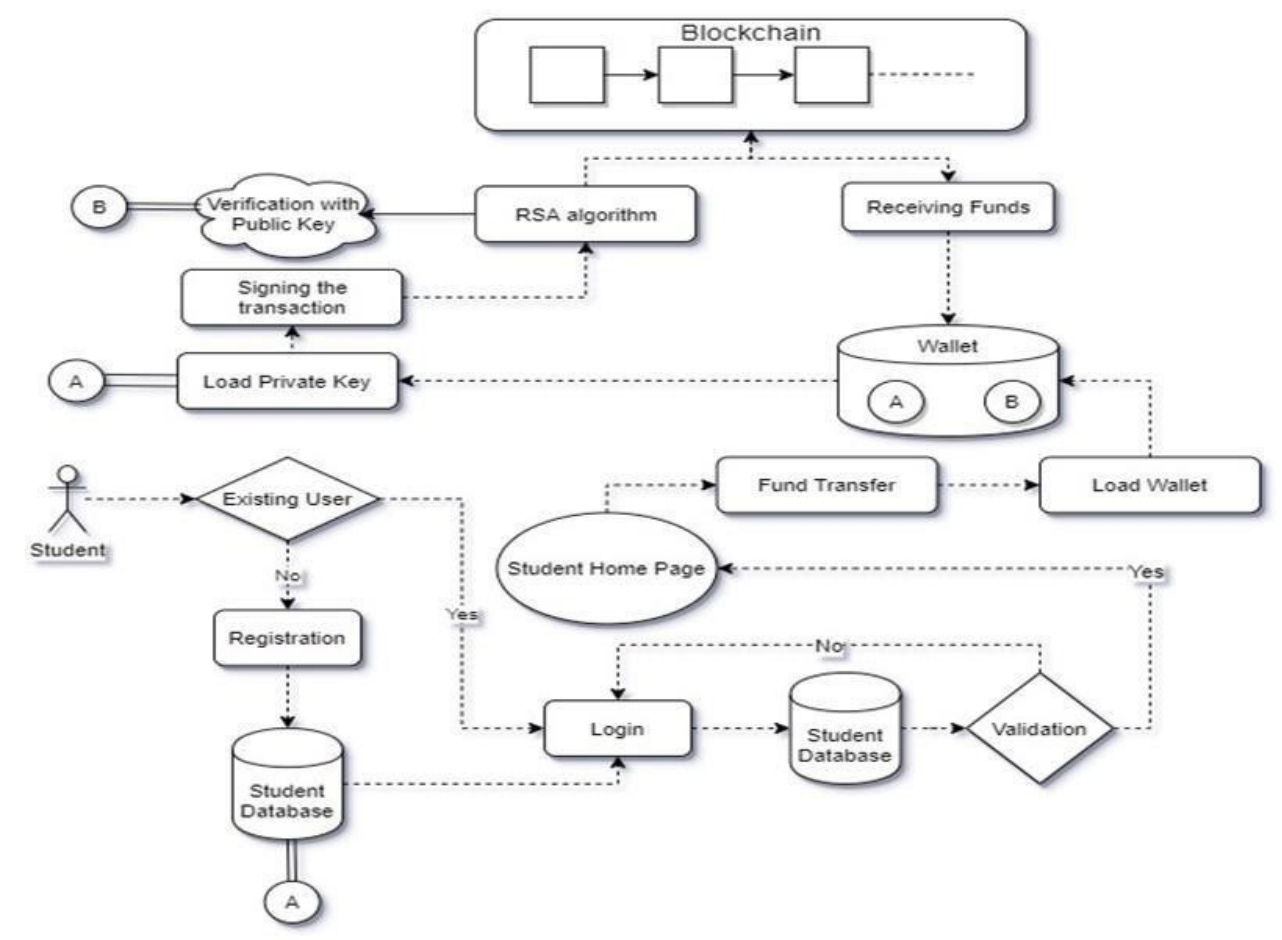

Figure 6. Private Cryptocurrency blueprint

\section{Conclusion}

After considering the many diverse reasons for unsatisfactory student performance and identifying the lack of intrinsic motivation as the root cause the paper proposes a new educational model. The model uses money as a tangible reward and uses cryptocurrency to carry the transactions. The paper also discusses the root concepts and strategies used by the model to alleviate probability of success. The paper further gives a blueprint to implement the model with either public cryptocurrency like Ethereum or via private cryptocurrency.

The paper focuses a lot on implementation of the model in educational institutes. The concept however can be expanded and applied across a wide array of domains ranging from Cibil score of employees in HR systems to determine appraisal, loyalty programs in gymnasiums, to an incentive system for employees in sales/marketing/trading based jobs.

\section{References}

[1] Samanez-Larkin, G.R. and Knutson, B., Decision making in the ageing brain: changes in affective and motivational circuits. Nature Reviews Neuroscience, 16(5), p.278.(2015)

[2] Worthy, D.A., Cooper, J.A., Byrne, K.A., Gorlick, M.A. and Maddox, W.T., State-based versus rewardbased motivation in younger and older adults. Cognitive, Affective, Behavioral Neuroscience, 14(4), pp.1208-1220.(2014)
[3] Eppinger, B., Walter, M., Heekeren, H.R. and Li, S.C., Of goals and habits: agerelated and individual differences in goal-directed decision-making. Frontiers in neuroscience, 7, p.253.(2013)

[4] Wirth, C., Akrour, R., Neumann, G. and Furnkranz, J.,A survey of preference- based reinforcement learning methods. The Journal of Machine Learning Research, 18(1), pp.4945-4990.(2017)

[5] McGraw, K.O., 1978. The detrimental effects of reward on performance: A literature review and a prediction model. The hidden costs of reward: New perspectives on the psychology of human motivation, pp.33-60.

[6] Ellingsen, T. and Johannesson, M. ,Pride and prejudice: The human side of incentive theory. American economic review, 98(3), pp.990- 1008.(2008)

[7] David R. Roalf, Suzanne H. Mitchell, William T. Harbaugh, Jeri S. Janowsky, Risk, Reward, and Economic Decision Making in Aging, The Journals of Gerontology: Series B, 67B, Issue 3, Pages 289-298.(2012)

[8] Seward, J.P., Drive, incentive, and reinforcement. Psychological review, 63(3), p.195.(1956)

[9] Brewer, E.W., Dunn, J.O. and Olszewski, P., Extrinsic reward and intrinsic motivation: The vital link between classroom management and student performance. Journal of Education for Teaching, 14(2), pp.151170.(1988)

[10] Carlozo, L., What is blockchain?. Journal of Accountancy, 224(1), p.29.(2017)

[11] Lemieux, V.L., Trusting records: is Blockchain technology the answer?. Records Management Journal,26(2), pp.110-139.(2016) 
[12] Delmolino, K., Arnett, M., Kosba, A., Miller, A. and Shi, E., February. Step by step towards creating a safe smart contract: Lessons and insights from a cryptocurrency lab. In International Conference on Financial Cryptography and Data Security (pp. 79- 94). Springer, Berlin, Heidelberg (2016)

[13] Teutsch, J., Jain, S. and Saxena, P., February. When cryptocurrencies mine their own business. In Inter- national Conference on Financial Cryptography and Data Security (pp. 499-514). Springer, Berlin, Heidelberg.(2016)

[14] Stefansson, G. and Lentin, J., From Smileys to Smileycoins:Using a Cryptocurrency in Education. Ledger, 2, pp.38-54.(2017) 